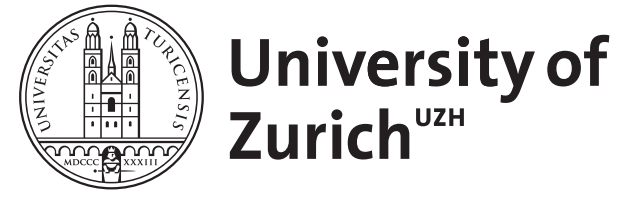

\title{
Zytologische Sarkomdiagnostik
}

\author{
Bode-Lesniewska, B
}

\begin{abstract}
Soft tissue swelling represents a common clinical sign of a variety of neoplastic and nonneoplastic lesions. Sarcoma is rarely a cause. Fine needle biopsy as a minimally invasive, economic and accurate method is well suited for the diagnosis of inflammatory/infectious processes as well as of recurrent and metastatic disease. Cytologic diagnosis of primary soft tissue tumors is also feasible. It requires close collaboration with other medical disciplines and incorporation of clinical, radiological and morphologic findings. Clinical data such as age, gender, size and topography are important parameters. The differentiation of cells and properties of the extracellular matrix supplies clues for the differential diagnosis and forms the starting point for immunohistochemical or molecular analysis (FISH, RT-PCR). This analysis may be performed on cytological smears, paraffin embedded material of the cell blocks or on frozen material.
\end{abstract}

DOI: https://doi.org/10.1007/s00292-010-1396-9

Other titles: Cytologic diagnosis of sarcoma

Posted at the Zurich Open Repository and Archive, University of Zurich

ZORA URL: https://doi.org/10.5167/uzh-50729

Journal Article

Published Version

Originally published at:

Bode-Lesniewska, B (2011). Zytologische Sarkomdiagnostik. Der Pathologe, 32(1):14-23.

DOI: https://doi.org/10.1007/s00292-010-1396-9 

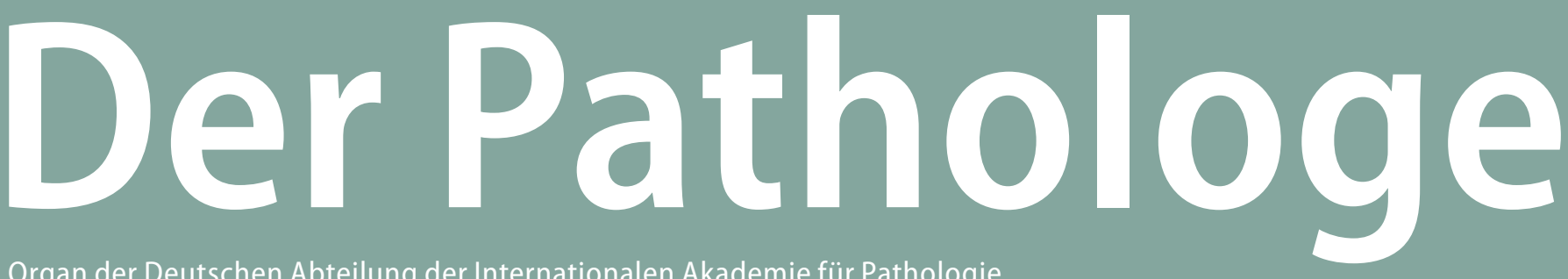

Organ der Deutschen Abteilung der Internationalen Akademie für Pathologie, der Deutschen, der Österreichischen und der Schweizerischen Gesellschaft für Pathologie und des Berufsverbandes Deutscher Pathologen

\section{Elektronischer Sonderdruck für}

\section{B. Bode-Lesniewska}

Ein Service von Springer Medizin

\section{B. Bode-Lesniewska}

\section{Zytologische Sarkomdiagnostik}


Pathologe 2011 · 32:14-23

DOI 10.1007/s00292-010-1396-9

Online publiziert: 31. Oktober 2010

c) Springer-Verlag 2010

\section{Schwerpunktherausgeber}

R. Büttner, Bonn

\section{B. Bode-Lesniewska}

Institut für Klinische Pathologie, UniversitätsSpital Zürich, Schweiz

\section{Zytologische Sarkomdiagnostik}

gen (Immunhistochemie, Fluoreszenzin-situ-Hybridisierung/FISH, Polymerasekettenreaktion/PCR) möglich, sollte eine genaue Aussage über die Linienzugehörigkeit und Dignität der Neoplasie angestrebt werden.

Über beeindruckende Ergebnisse der FNP bei Weichteilneoplasien wird vor allem aus Schweden (Lund; [5-7]) und Frankreich (Institut Curie, Paris; [2, 1822]) berichtet. In diesen Zentren ist die FNP bei Verdacht auf einen Weichteiltumor ein integrales Element der multidisziplinären Abklärungsuntersuchungen. Die technischen Fortschritte der letzten Jahre machen die Übernahme der diagnostischen und prognostischen Zusatzuntersuchungen der Histologie (Immunhistochemie, FISH, Reverse-Transkriptase-/RTPCR, Sequenzierung) und ihre Anwendung an den zytologischen Proben selbstverständlich. Das Vorgehen folgt der derzeitigen allgemeinen Tendenz in Richtung minimal-invasiver Abklärungsuntersuchungen und erfüllt die gesundheitspolitische Forderung, Kosten ohne Qualitätseinbuße zu minimieren. Die in der Regel mit kaum oder nur minimalen Komplikationen behaftete FNP stellt durchaus eine technisch wenig anspruchsvolle, ambulante Alternative dar im Vergleich zu einer offenen Biopsie mit deutlich größeren Risiken im Zusammenhang mit der Anästhesie, Nachblutung, Infektion oder Kontamination des Stichkanals. Kosteneffizienz und schnellere Verarbeitungszeiten gehören zu den weiteren wichtigen Vorteilen der FNP [1, 13, 14, 23-25, 28].

In den Händen erfahrener Zytologen erreichen Sensitivität und Spezifität der FNP in Bezug auf die Unterscheidung benigner von malignen Läsionen nahezu 95\%. Die „falsch-positive“ Rate wird in der Literatur mit 0-5\%, die „falsch-negative" Rate mit 2-15\% angegeben [12, 25]. Die „falsch-negativen“ Diagnosen können meistens auf suboptimales Material oder Fehlinterpretationen zurückgeführt werden und sind in der Regel nicht mit schwerwiegenden Folgen behaftet, da eine klinisch suspekte Läsion ohnehin weiter morphologisch abgeklärt wird.

\section{Entnahmetechnik und Probenverarbeitung}

Grundlegend entscheidend für den diagnostischen Erfolg einer FNP (selbstverständlich nicht nur auf dem Gebiet der Weichteilpathologie) ist die korrekte Entnahmetechnik. Ähnlich wie bei der Auswahl der Biopsiestelle an den Extremitäten, muss der transkutane FNP-Stichkanal unter Vermeidung einer eventuellen Kontamination der nicht vom Tumor betroffenen Kompartimente angelegt werden. In der Regel bedeutet dies, dass der kürzeste Weg zwischen dem Tumor und der Haut - am besten in der Absprache mit dem beteiligten Chirurgen/Orthopäden - gewählt wird. Die Punktion wird in der Regel mit einer dünnen Nadel (22-25 Gauge) durchgeführt, von Vorteil ist der Gebrauch eines Spritzenhalters (z. B. Ca$\left.\operatorname{meco}^{\circledR}\right)$. Das Material wird fächerförmig (um die Materialentnahme aus möglichst großem Tumorvolumen zu gewährleisten) mit schneidenden Bewegungen unter dem nach Bedarf angelegten Sog über etwa 20-30 s gewonnen.

Die bewusste Materialasservierung in entsprechenden Medien für diagnostische 
Zusatzmethoden ist von entscheidender Bedeutung. Dieses Ziel lässt sich erfahrungsgemäß am besten erreichen, wenn der Eingriff entweder vom Zytopathologen selbst, häufig unter Ultraschallsteuerung, durchgeführt wird (die in unserem Institut bevorzugte Variante) oder wenn die Punktion in Anwesenheit eines Zytologen oder eines speziell ausgebildeten Zytotechnikers erfolgt.

Bei intrathorakal oder intraabdominell gelegenen Tumoren muss eine Computertomographie- (CT-)gesteuerte transkutane oder transendoskopische ultraschallgesteuerte FNP durchgeführt werden. Häufig, besonders bei kollagenarmen, zellreichen Läsionen gelingt die Entnahme von ausreichenden Gewebemengen bereits in Rahmen einer einzigen Punktion. Bei Bedarf lassen sich in der gleichen Sitzung weitere Punktionen durchführen die FNP ist für den Patienten wenig belastend. Eine Lokalanästhesie ist bei oberflächlichen, d. h. ohne CT-Steuerung erreichbaren Knoten nicht notwendig. Primär werden 3 bis 5 Direktausstriche angefertigt, die z. T. alkoholfixiert und $z$. T. luftgetrocknet werden.

Die feucht fixierten Ausstriche werden nach Papanicolaou, die luftgetrockneten mit Diff-Quick gefärbt. Die Papanicolaou-Färbung erlaubt eine bessere Beurteilung der Kerneigenschaften, während die Diff-Quick-Methode die Eigen- schaften der extrazellulären Grundsubstanz hervorhebt. Mittels Diff-QuickFärbung (Zeitaufwand weniger als $1 \mathrm{~min}$ ) lässt sich zusätzlich schnell beurteilen, ob ausreichend diagnostisches Material erfasst wurde. Die Direktausstriche eignen sich sehr gut für FISH-Untersuchungen, da bei dreidimensional erhaltenen Zellen das durch Schneiden der Kerne entstehende histologische Artefakt in der Anzahl der Signale entfällt (• Abb. 4 d, - Abb. $5 \mathrm{~d}$ ).

Das Restmaterial des Punktats wird für die Herstellung eines formalinfixierten, paraffineingebetteten Zellblocks (- Abb. 1 d, e, $\bullet$ Abb. 3 c-e, $\bullet$ Abb. 4 c, - Abb. 5 c) eingesetzt, wobei die Verwendung von hämolysierenden Flüssigkeiten (z. B. Cytolyt ${ }^{\circledast}$, Cytyc $^{\circledast}$ ) von Vorteil ist. Die Anwendung von Dünnschichtmethoden in der Zytologie der Weichteilneoplasien wird nicht empfohlen, weil damit besonders bei Weichteiltumoren wichtige Details des Tumoraufbaus und die extrazelluläre Grundsubstanz verloren gehen [3]. Ein Teil des Punktionsmaterials kann nach Bedarf schockgefroren oder für die Tumorzytogenetik gewonnen werden [15].

\section{Zytomorphologische Klassifikation}

Als Faustregel gilt in der Diagnostik einer Weichteilschwellung, einen entzündlichen
Prozess, eine Melanom-, Karzinom- oder Lymphommanifestation auszuschließen, ehe man einen primären Weichteiltumor in Erwägung zieht. Die Interpretation des Punktats eines primären Weichteiltumors wird durch eine strukturierte Vorgehensweise, die auf dem zytomorphologischen Grundmuster der Direktausstriche basiert, erleichtert $[1,23]$.

Verschiedene Autoren schlugen die Einordnung der Punktate in unterschiedliche zytoarchitektonische Gruppen vor, die sich aufgrund eigener Erfahrungen in der Praxis am besten in sechs Kategorien zusammenfassen lassen (• Tab. 1, [12, 17, 23]).

Die große Spannbreite in der morphologischen Differenzierung einzelner Entitäten der Weichteilneoplasien führt dazu, dass der gleiche Tumortyp in der Differenzialdiagnose mehr als einem Grundmuster zugeordnet werden kann wie z. B. das Neurofibrom (myxoides oder spindelzelliges Grundmuster) oder das Synovialsarkom (spindelzelliges, rundzelliges oder epitheloides Grundmuster). Die an den Direktausstrichen erhobenen Befunde werden mit den Befunden an „Mikro-Stanzbiopsien“" in Zellblockpräparaten verglichen (- Abb. $1 \mathrm{~d}, \mathrm{e}, \bullet$ Abb. $3 \mathrm{c}-\mathrm{e}$, - Abb. 4 c, - Abb. 5 c). Beides, ergänzt durch die Ergebnisse der Zusatzuntersuchungen fließt in die Endbeurteilung mit ein.

\section{Hier steht eine Anzeige.}



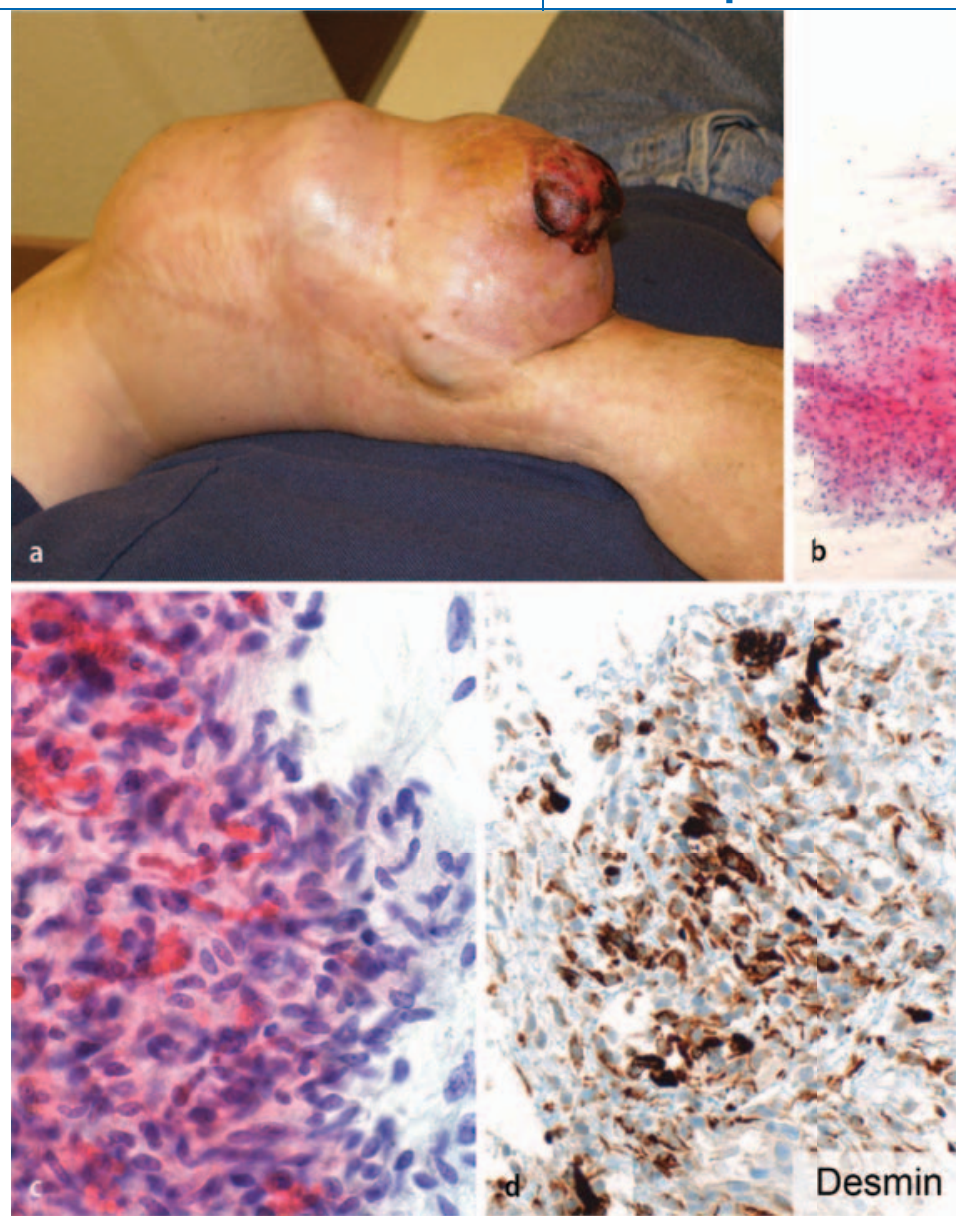

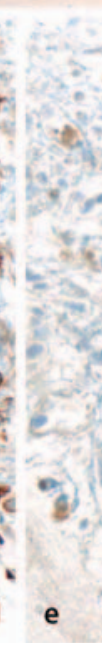

e

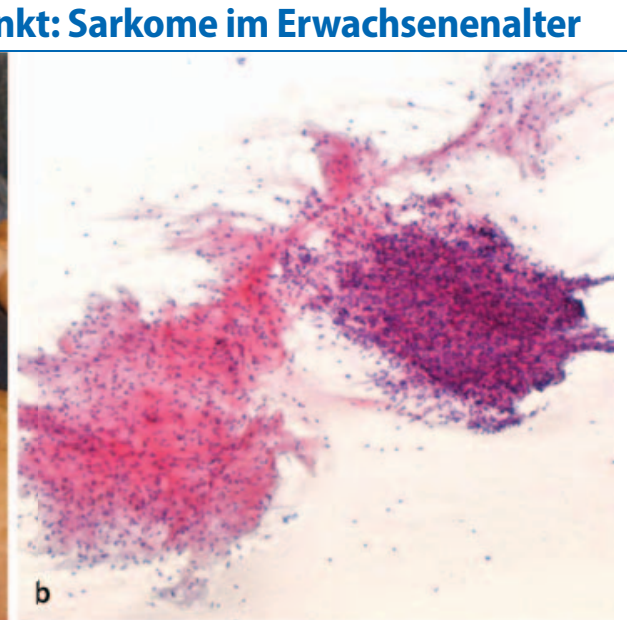

Abb. $1<$ a 47-jähriger Mann mit einem exulzerierten, schnell über wenige Monate wachsenden Tumor am linken Unterarm. b Die Direktausstriche der Feinnadelpunktion zeigten eine wechselnde Zelldichte der mesenchymalen Neoplasie (PapanicolaouFärbung, Vergr. 50:1). c Die Tumorzellen sind monomorph, spindelig mit teils dichteren eosinophilen, exzentrischen Zytoplasmasäumen (PapanicolaouFärbung, 400:1). Im Material des Zellblocks fand sich immunhistochemisch die Expression von (d; 200:1) und Myogenin (e; 200:1). Bei fehlenden Zeichen der Neurofibromatose und ohne Zusammenhang mit großen Nerven wurde die Diagnose eines SpindelzellRhabdomyosarkoms bevorzugt. Diese Diagnose wurde im Amputat bestätigt

\section{Zytologische Grundmuster primärer Weichteilneoplasien}

\section{Spindelzellen}

Das häufigste morphologische Bild bei zytologischen Untersuchungen primärer Weichteilläsionen ist eine spindelige Zellpopulation unterschiedlicher Zelldichte und Atypiegrades. Diese zytoarchitektonische Gruppe ist die umfangreichste Kategorie mit ausgesprochener Vielfalt der differenzialdiagnostisch infrage kommenden Läsionen. Die Differenzialdiagnose umfasst nicht nur sekundäre oder primäre, benigne und maligne Weichteilneoplasien, sondern auch reaktive bzw. pseudosarkomatöse Prozesse. Die klinische und bildgebende Korrelation wie auch die Anwendung der diagnostischen Zusatzmethoden ist in dieser Kategorie äußerst wichtig, da Läsionen aus dieser Gruppe mit einem hohen Risiko der Fehlbeurteilung behaftet sind [12].

Allgemein gilt, dass benigne Tumoren eher zellarme Punktate mit kompak- ten Gruppen von kleinen, monomorphen Zellen ergeben, während das Material maligner Neoplasien zahlreiche diskohäsive, pleomorphe und mitotisch aktive Zellen enthält.

Der topographische Zusammenhang mit größeren Nerven lenkt die differenzialdiagnostischen Überlegungen $\mathrm{zu}$ den peripheren Nervenscheidentumoren, während eine intraabdominelle Lokalisation die Diagnose eines gastrointestinalen Stromatumors (GIST) favorisieren lässt. Eine periartikuläre Lokalisation eines Tumors bei einem jungen Patienten macht die Diagnose eines Synovial sarkoms wahrscheinlich, während bei einer großen, tief liegenden Raumforderung am Oberschenkel eines älteren Patienten ein dedifferenziertes Liposarkom bzw. ein Leiomyosarkom infrage kommt.

Starke immunhistochemische Positivität für S1oo bei einem spindelzelligen Tumor (nach Ausschluss einer Melanommanifestation) ist bei den nichtepitheloiden malignen peripheren Nervenscheidentumoren (MPNST) ungewöhnlich und spricht für einen benignen neurogenen Tumor. Die immunzyto- bzw. immunhistochemisch nachgewiesene Expression der myogenen Marker (Aktin, Desmin, Myogenin, MyoD1) lässt eine leiomyomatöse bzw. rhabdomyomatöse (• Abb. 1 d, e) Differenzierung vermuten. Die Expression von CD117 im entsprechenden klinischen Kontext belegt die Diagnose eines GIST, während der Nachweis einer SYT-SSX-Translokation für die Diagnose eines Synovialsarkoms beweisend ist (• Tab. 2).

\section{Myxoide Grundsubstanz}

Die Erkennung dieser zytomorphologischen Kategorie basiert nicht auf den vorhandenen Zellen, sondern auf der reichlich im Hintergrund vorliegenden myxoiden Grundsubstanz, die sich charakteristisch in der Papanicolaou-Färbung amorph grünlich bis blau darstellen lässt $[16,31]$. Die Differenzialdiagnose umfasst einige Entitäten, deren Unterscheidung aufgrund der in der Grundsubstanz ein- 
gebetten Zellen unter Berücksichtigung der klinischen und bildgebenden $\mathrm{Zu}$ sammenhänge erfolgt. Praktisch azelluläre mukoide Grundsubstanz ist bei einem Ganglioninhalt zu erwarten, während spärliche, blande, kleine Spindelzellen ein intramuskuläres oder juxtaartikuläres Myxom bevorzugen lassen. Auffallend verzweigte, zarte Kapillaren auf myxoidem Hintergrund begleitet von perivaskulär betonten, monomorphen, ovalären, kleinen Zellen in einer tief liegenden Läsion am Oberschenkel lassen die Diagnose eines myxoiden Liposarkoms vermuten (- Abb. 2). Diese Verdachtsdiagnose lässt sich durch den mittels FISH bzw. RT-PCR erfolgten Nachweis der diagnostischen Translokation (• Tab. 2; - Abb. 2 d) bestätigen.

Im Gegensatz zum myxoiden Liposarkom zeigt das myxoide (extraskelettale) Chondrosarkom eine charakteristische, leuchtend rote, metachrome Anfärbbarkeit in einer Diff-Quick-Färbung am luftgetrockneten Ausstrich. Die in der Grundsubstanz eingelagerten Zellen sind ebenfalls eher monomorph und bland. Im Gegensatz dazu zeigt das Myxofibrosarkom eine auffallende Pleomorphie der auf einem myxoiden Hintergrund sichtbaren Zellen. In der Studie von Kilpatrick [16] konnte zytologisch in 13 von 16 (81\%) Patienten mit myxoiden Sarkomen der genaue Sarkomtyp bestimmt werden.

\section{Pleomorphe Zellen}

Die Anwesenheit von zahlreichen, meist großen und unregelmäßig gestalteten Zellen lässt eine Weichteilschwellung in die Gruppe der pleomorphzelligen Läsionen einordnen. Dieses Grundmuster ist meist im Untersuchungsmateriel von älteren Patienten $\mathrm{zu}$ erwarten und wirft eine relativ breite Differenzialdiagnose von überwiegend hoch malignen Tumoren auf. Als erstes gilt es, eine Manifestation eines wenig differenzierten (großzelligen) Karzinoms, eines malignen Melanoms bzw. eines anaplastischen großzelligen Lymphoms auszuschließen, was in der Regel in der Korrelation mit der Klinik und durch den Einsatz von Antikörpern gegen Zytokeratine, melanozytäre (S1oo, HMB45, Melan A) und lymphatische (CD45, CD3o, ALK1, CD2O, CD 3 )
Pathologe 2011 · 32:14-23 DOI 10.1007/s00292-010-1396-9

(c) Springer-Verlag 2010

\section{B. Bode-Lesniewska}

Zytologische Sarkomdiagnostik

\section{Zusammenfassung}

Weichteilschwellungen sind das Symptom einer Vielzahl neoplastischer und nichtneoplastischer Veränderungen. Sarkome gehören zu den selteneren Ursachen. Die Feinnadelpunktion hat sich in der Abgrenzung zu entzündlichen Prozessen sowie in der Abklärung von Tumorrezidiven und Karzinommetastasen als minimal-invasive, kostengünstige und zuverlässige Methode bewährt. Auch in der Abklärung von Weichteiltumoren kann sie zu einer präzisen Diagnose führen. Voraussetzung ist eine enge multidisziplinäre Zusammenarbeit unter Einbeziehung klinischer, radiologischer und morphologischer Befunde. Für die zytologische Befundung sind Alter und Geschlecht sowie Topographie, Größe und Wachstumsgeschwindigkeit

\section{Cytologic diagnosis of sarcoma}

\section{Abstract}

Soft tissue swelling represents a common clinical sign of a variety of neoplastic and non-neoplastic lesions. Sarcoma is rarely a cause. Fine needle biopsy as a minimally invasive, economic and accurate method is well suited for the diagnosis of inflammatory/infectious processes as well as of recurrent and metastatic disease. Cytologic diagnosis of primary soft tissue tumors is also feasible. It requires close collaboration with other medical disciplines and incorporation of clinical, radiological and morphologic findings. Clinical data such as age, gender, size and topography are important parameters. The differ- des Tumors wichtige Parameter. Reifungsgrad und Form der Zellen sowie Vorhandensein und Ausdifferenzierung der bindegewebigen Matrix bieten erste differenzialdiagnostische Hinweise und sind der Ausgangspunkt für immunzytochemische und molekularbiologische (FISH, RT-PCR) Zusatzuntersuchungen. Diese Untersuchungen werden an Direktausstrichen, an mittels Zellblockmethode eingebettetem und an tiefgefrorenem Punktionsmaterial ausgeführt.

\section{Schlüsselwörter}

Feinnadelpunktion - Weichteiltumor . Sarkom · Immunhistochemie · Fluoreszenzin-situ-Hybridisierung

entiation of cells and properties of the extracellular matrix supplies clues for the differential diagnosis and forms the starting point for immunohistochemical or molecular analysis (FISH, RT-PCR). This analysis may be performed on cytological smears, paraffin embedded material of the cell blocks or on frozen material.

\section{Keywords}

Fine needle biopsy - Soft tissue tumor . Sarcoma $\cdot$ Immunohistochemistry . Fluorescence in situ hybridization 
Tab. 1 Zytoarchitektonische Grundmuster der Feinnadelpunktate der primären Weichteiltumoren

\begin{tabular}{|c|c|c|c|c|c|}
\hline Spindelzellen & Myoxide Matrix & Pleomorphe Zellen & Runde Zellen & Epitheloide Zellen & $\begin{array}{l}\text { Differenziertes } \\
\text { Gewebe }\end{array}$ \\
\hline \multicolumn{6}{|c|}{ Gutartige, nichtmetastasierende Tumoren } \\
\hline $\begin{array}{l}\text { Neurofibrom } \\
\text { Schwannom } \\
\text { Noduläre Fasziitis } \\
\text { Proliferative Fasziitis } \\
\text { Proliferative Myositis } \\
\text { Tenosynovialer Riesenzell- } \\
\text { tumor } \\
\text { Leiomyom } \\
\text { Fibromatose (Desmoidtumor) }\end{array}$ & $\begin{array}{l}\text { Ganglion, Bursa } \\
\text { Myxom } \\
\text { - intramuskuläres } \\
\text { - juxtaartikuläres } \\
\text { Noduläre Fasziitis } \\
\text { Neurofibrom } \\
\text { Spindelzelllipom } \\
\text { Lipoblastom }\end{array}$ & $\begin{array}{l}\text { Pleomorphes Lipom } \\
\text { Proliferative Myositis } \\
\text { Proliferative Fasziitis }\end{array}$ & - & $\begin{array}{l}\text { Granularzelltumor } \\
\text { Proliferative Myositis }\end{array}$ & $\begin{array}{l}\text { Lipom } \\
\text { Chondrom }\end{array}$ \\
\hline \multicolumn{6}{|c|}{ Läsionen mit morphologisch schwer einschätzbarem Metastasierungspotenzial } \\
\hline $\begin{array}{l}\text { Solitärer fibröser Tumor } \\
\text { GIST } \\
\text { DFSP }\end{array}$ & - & Paragangliom & - & $\begin{array}{l}\text { Paragangliom } \\
\text { Epitheloides Häm- } \\
\text { angioendotheliom }\end{array}$ & - \\
\hline \multicolumn{6}{|l|}{ Eindeutig maligne Neoplasien } \\
\hline $\begin{array}{l}\text { MPNST } \\
\text { Synovialsarkom } \\
\text { Leiomyosarkom } \\
\text { Dedifferenziertes Liposarkom } \\
\text { Myofibroblastisches Sarkom } \\
\text { Embryonales Rhabdomyo- } \\
\text { sarkom } \\
\text { Kaposi-Sarkom }\end{array}$ & $\begin{array}{l}\text { Myxoides Liposar- } \\
\text { kom } \\
\text { Myxofibrosarkom } \\
\text { Myxoides Chon- } \\
\text { drosarkom } \\
\text { Niedrig malignes } \\
\text { fibromyxoides } \\
\text { Sarkom }\end{array}$ & $\begin{array}{l}\text { Undifferenziertes pleo- } \\
\text { morphes Sarkom } \\
\text { Pleomorphes Leiomyo- } \\
\text { sarkom } \\
\text { Pleomorphes Rhabdo- } \\
\text { myosarkom } \\
\text { Pleomorphes Liposarkom } \\
\text { Pleomorphes MPNST } \\
\text { Angiosarkom } \\
\text { Extraskelettales Osteo- } \\
\text { sarkom }\end{array}$ & $\begin{array}{l}\text { Alveoläres Rhabdomyosarkom } \\
\text { Neuroblastom } \\
\text { Ewing-Sarkom/PNET } \\
\text { Synovialsarkom } \\
\text { Rundzelliges Liposarkom } \\
\text { Mesenchymales Chondro- } \\
\text { sarkom } \\
\text { DSRCT } \\
\text { Rhabdoidtumor } \\
\text { Pigmentierter, neuroektoder- } \\
\text { maler Tumor des Kinderalters }\end{array}$ & $\begin{array}{l}\text { Epitheloides Sarkom } \\
\text { Epitheloides Angio- } \\
\text { sarkom } \\
\text { Epitheloides Leio- } \\
\text { myosarkom } \\
\text { Epitheloides MPNST } \\
\text { Synovialsarkom } \\
\text { Alveoläres Weichteil- } \\
\text { sarkom } \\
\text { Klarzellsarkom } \\
\text { Rhabdoidtumor }\end{array}$ & $\begin{array}{l}\text { Gut differenzier- } \\
\text { tes Lipo- } \\
\text { sarkom } \\
\text { Niedrig malignes } \\
\text { Chondrosarkom }\end{array}$ \\
\hline
\end{tabular}

Grundregel: Zuerst eine Manifestation eines Karzinoms, Melanoms, Lymphoms, Mesothelioms ausschließen! GIST gastrointestinaler Stromatumor, DFSP Dermatofibrosarcoma protuberans, MPNST maligner peripherer Nervenscheidentumor, PNET primitiver neuroektodermaler Tumor, DSRCT desmopolastischer klein-, blau- und rundzelliger Tumor.

Tab. 2 Wichtige Weichteilsarkome mit diagnostisch relevanten zytogenetischen Veränderungen

\section{Tumor}

Ewing-Sarkom/PNET Zytogenetische Aberration

Alveoläres Rhabdomyosarkom

$t(11 ; 22)$

$\mathrm{t}(21 ; 22)$

$\mathrm{t}(2 ; 13)$

$\mathrm{t}(1 ; 13)$

Myxoides/rundzelliges Liposarkom

$\mathrm{t}(12 ; 16)$

$t(12 ; 22)$

Myxoides extraskelettales Chondrosarkom

$\mathrm{t}(9 ; 22)$

$\mathrm{t}(9 ; 17)$

$\mathrm{t}(9 ; 15)$

Synovialsarkom

$t(X ; 18)$

Dermatofibrosarcoma protuberans

$t(17 ; 22)$

$t(12 ; 15)$

Kongenitales Fibrosarkom

$t(11 ; 22)$

Desmoplastischer klein- und rundzelliger Tumor

Alveoläres Weichteilsarkom

$\mathrm{t}(\mathrm{X} ; 17)$

Klarzellsarkom

$\mathrm{t}(12 ; 22)$

$\mathrm{t}(2 ; 22)$

Fibromyxoides Sarkom

$\mathrm{t}(7 ; 16)$

$\mathrm{t}(11 ; 16)$
Marker zu erreichen ist. Insbesondere bei Tumoren des Oberschenkels oder des Retroperitoneums muss man an primäre Weichteilsarkome denken, und zwar in erster Linie an ein dedifferenziertes Liposarkom mit hochgradig maligner sarkomatöser Komponente (Positivität für MDM2 und/oder CDK4). Parallel kommt auch ein myogenes Sarkom vom Typ eines hoch malignen Leiomyosarkoms (Positivität für Aktin und/oder Desmin) oder ein pleomorphzelliges Rhabdomyosarkom (Positivität für Desmin, Myogenin, MyoD1) infrage. Weitere differenzialdiagnostische Optionen in dieser Gruppe stellen das pleomorphzellige Liposarkom (bizarre, pleomorphe Lipoblasten), das extraskelettale Osteosarkom (Tumorosteoid), das Angiosarkom (Positivität für $\mathrm{CD}_{31}$ und/oder $\mathrm{CD}_{34}$ ) oder der MPNST [auf das Vorliegen einer Neurofibromatose hinweisende Vorgeschichte (• Abb. 3) bzw. bildgebend festgestellter Zusammenhang mit größeren Nerven] dar.

Trotz des Einsatzes aller verfügbaren Hilfsmethoden verbleibt eine Subgrup- 
Abb. 2 - a Der 39-jährige Mann bemerkte einen über 2 Jahre langsam wachsenden Knoten am rechten Oberschenkel. b Die MRT zeigte einen großen intramuskulären Tumor. c Die FNP ergab eine myxoide Neoplasie mit kleinen monomorphen Zellen und prominentem Geflecht zarter kapillärer Blutgefäße, entsprechend einem myxoiden Liposarkom ( $\mathrm{Pa}$ panicolaou-Färbung, Vergr. 200:1). d Die RT-PCR bestätigte das Vorliegen des diagnostischen Fusionsproduktes der Translokation $\mathrm{t}(12 ; 16)$ im Punktionsmaterial (FUS/DDIT3; Typ 3)

Abb. 3 - a Über 4 Monate schnell wachsender intramuskulärer Oberschenkeltumor rechts bei einem 53-jährigen Mann (MRT). Vor 2 Jahren wurde bei inm ein plexiformes Neurofibrom temporal rechts entfernt. b Die FNP des Oberschenkeltumors ergab eine partiell nekrotische, pleomorphzellige Neoplasie (Papanicolaou-Färbung, Vergr. 400:1), teils mit exzentrischen, dichten Zytoplasmasäumen (Inset: Zellblock; HE-Färbung, 630:1). Immunhistochemisch wurde auf dem Material des Zellblocks ein hoher Proliferationsindex MIB-1 (c; 200:1) sowie eine herdförmige Expression von Desmin $(\mathbf{d} ; 400: 1)$ und Myogenin (e; 200:1) nachgewiesen. Die Diagnose eines malignen peripheren Nervenscheidentumors (MPNST) mit rhabdomyosarkomatöser Differenzierung (Triton-Tumor) wurde gestellt (und im Resektat bestätigt)
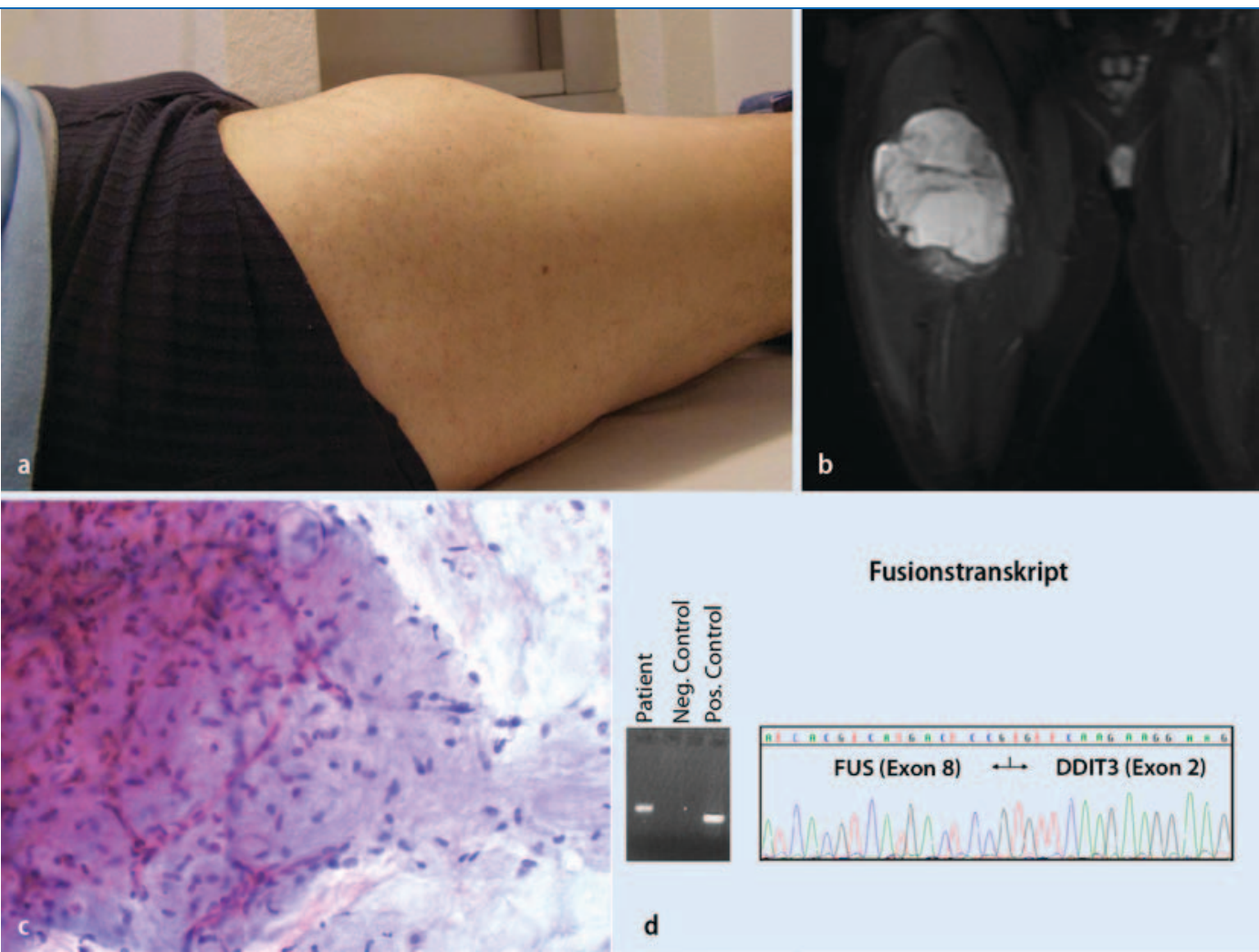

Fusionstranskript

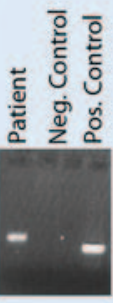

d
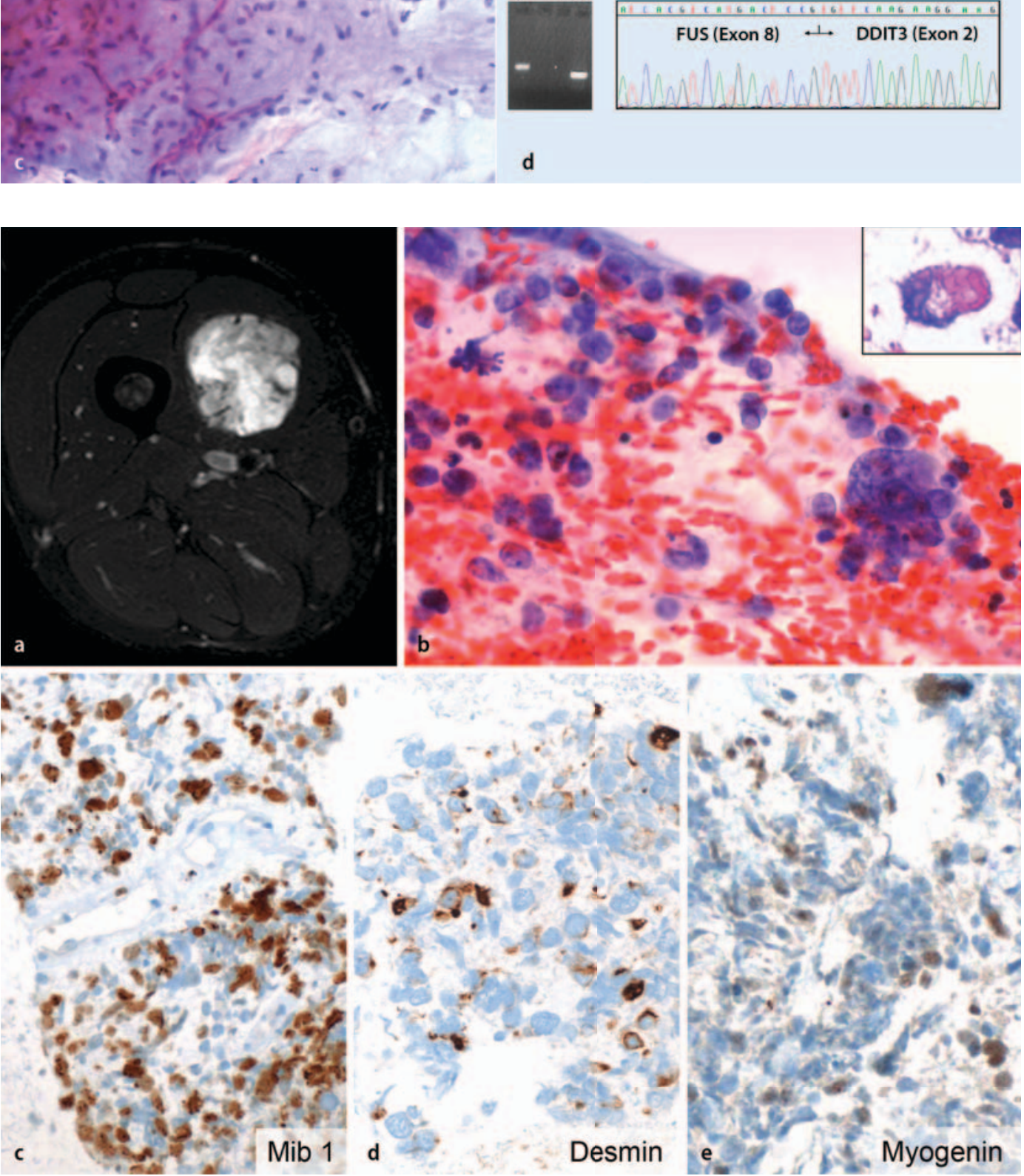

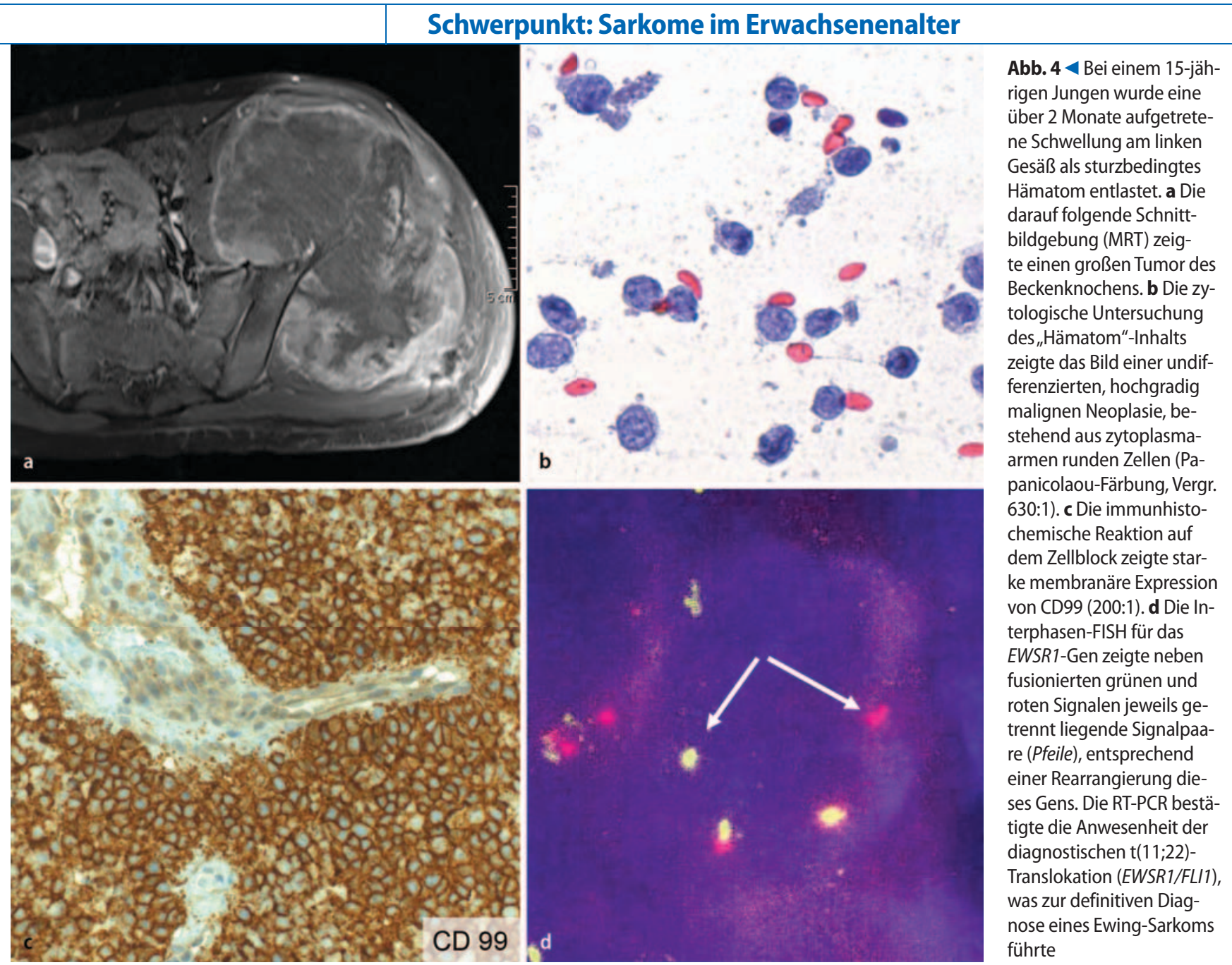

pe pleomorphzelliger Sarkome, die sich nicht weiter zuordnen lassen und gemäß der geltenden WHO-Klassifikation [9] als „undifferentiated high grade pleomorphic sarcoma" bezeichnet werden. Pragmatisch gesehen ist eine detaillierte Unterscheidung des Subtyps eines pleomorphzelligen, hoch malignen Sarkoms bei den zurzeit zur Verfügung stehenden therapeutischen Optionen für das klinische Management des Patienten nicht immer von prioritärer Bedeutung und auch nicht immer aufgrund der histologischen Untersuchung einer Stanzbiopsie zu erreichen. Solche Tumoren werden meistens primär operativ behandelt, sodass die definitive Subtypisierung am Resektat erfolgen kann.

\section{Runde Zellen}

Diese Gruppe umfasst hoch maligne Neoplasien vor allem des Kindesalters, die selten auch im Erwachsenenalter an- zutreffen sind. Insbesondere bei Erwachsenen muss man an Manifestationen der kleinzelligen Karzinome, Melanome oder (meist bereits vordiagnostizierten) Lymphome denken. In der Diagnostik der so genannten „klein-, blau- und rundzelligen " Tumoren ist der Einsatz der Zusatzmethoden in den meisten Fällen auch am histologischen Material unverzichtbar. Da es sich bei diesen Läsionen meist um sehr zellreiches Material handelt, enthalten sowohl die Direktausstriche als auch der Zellblock reichlich Tumorgewebe für immunhistochemische und molekulargenetische Untersuchungen. Sollten sich im Untersuchungsmaterial neben rundzelligen Anteilen weitere Bestandteile nachweisen lassen, so kann bei myxoidem Gewebe mit verzweigten zarten Kapillaren, die Diagnose eines rundzelligen Liposarkoms und bei chondroiden Anteilen ein mesenchymales Chondrosarkom vermutet werden.

Bereits der konventionell histologisch erfolgte Nachweis von Glykogen so- wie die Ergebnisse der Immunhistochemie für CD99, myogene Marker (Desmin, Myogenin, MyoD1), neuroendokrine Marker (Synaptophysin) und epitheliale Marker (Zytokeratin, EMA) können richtungsweisend für die Diagnose sein. Aufgrund des zytologischen Befunds und des Immunprofils lassen sich gezielte molekulargenetische Untersuchungen einsetzen ( $\bullet$ Tab. 2), die zur präzisen Diagnosestellung führen (• Abb. 4). Die Anwendung der Zusatzuntersuchungen bei den überwiegend aus runden Zellen bestehenden Weichteitumoren führt im höchsten Prozentsatz (bis zu 83\%) der Fälle im Vergleich mit anderen Grundmustern zu definitiver Subtypisierung [17].

\section{Epitheloide Zellen}

Große zytoplasmareiche, rund-ovaläre Zellen, die deutliche Zytoplasmagrenzen aufweisen und stellenweise kohäsive Zell- 

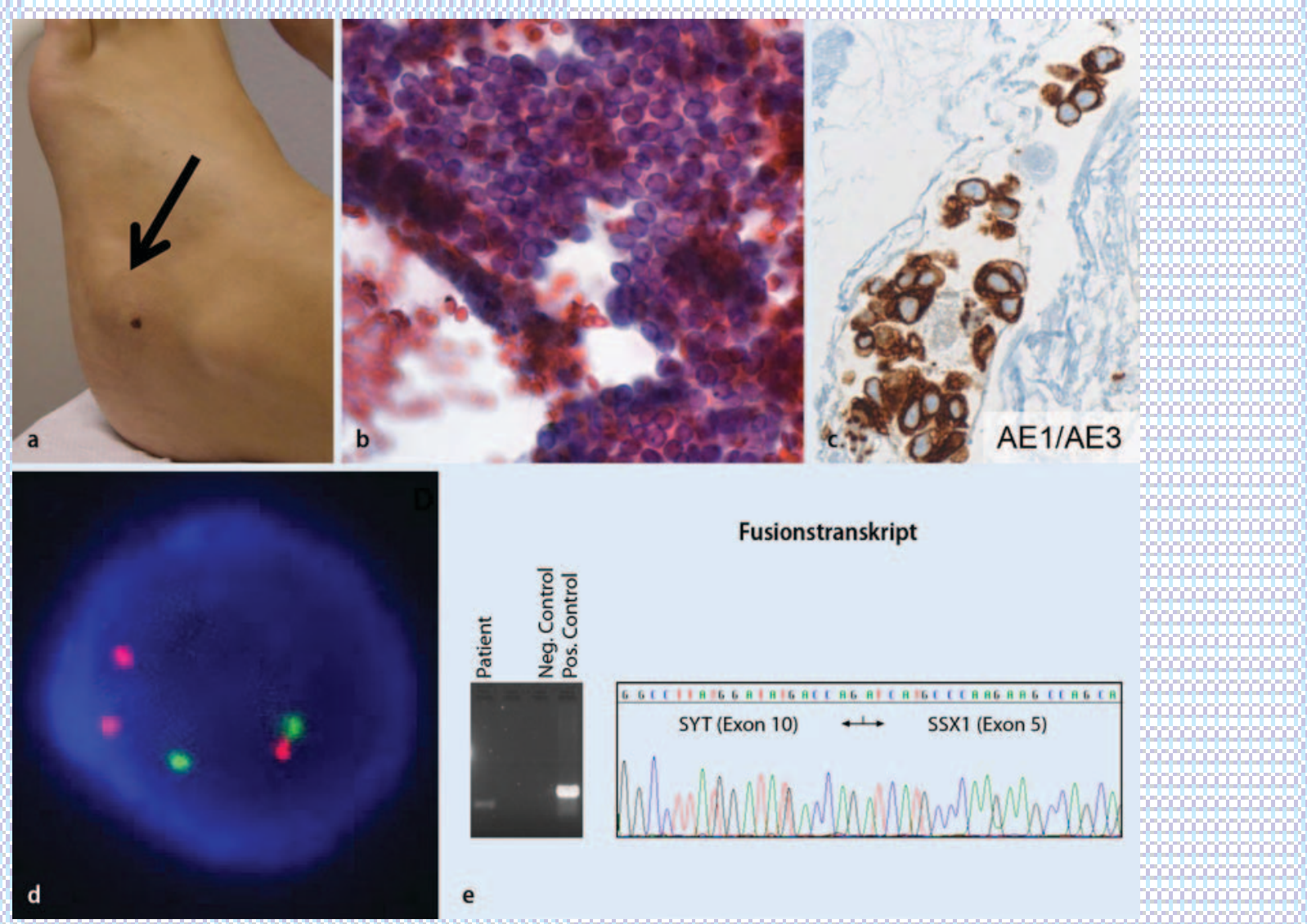

Abb. 5 L Langsam über 2 Jahre wachsender Tumor plantar links bei einer 27-jährigen Frau mit einem gemäßMRT ausgedehnten tokalen Tumorbefall. a Die lateral am linken Fuß gut tastbare und sichtbare Schwellung (Pfeil) wurde feinnadelpunktiert. Die Direktausstriche zeigten neben wenigen Spindelzellen, kohäsive „epitheliale“ Verbände monomorpher Zellen (b; $\mathrm{Pa}$ panicolaou-Färbung, Vergr. 400:1), welche immunhistochemisch im Material des Zellblocks eine starke Expression des Panzytokeratin-Markers AE1/AE3 zeigten (c; 400:1). d Die an den Direktausstrichen durchgeführte FISH für das SYT-Gen zeigte in allen Tumorzellkernen neben einem fusionierten Signal ein getrenntes Signalpaar sowie ein überzähliges rotes Signal, Dieses Ergebnis war suggestiv auf das Vorliegen einer Rearrangierung des SYT-Gens, assoziiert mit sekundären chromosomalen Anomalien. e Der Nachweis der $t(X ; 18)$-Translokation (SYT/SSX1) wurde mittels RT-PCR erbracht, und die Diagnose eines biphasischen Synovialsarkoms gestellt. Bei der Patientin wurden bereits bei Diagnosestellung multiple bilaterale Lungenmetastasen mittels CT diagnostiziert, und sie ist 2 Jahre später trotz adjuvanter Chemotherapie verstorben -

formationen bilden, werden bei einigen seltenen Weichteilneoplasien beobachtet. Es handelt sich dabei häufig um epitheloide Varianten von unterschiedlichen Sarkomsubtypen, wie z. B. biphasische bzw. überwiegend epitheliale Synovialsarkome (- Abb. 5), epitheloide Varianten eines Angiosarkoms oder eines MPNST, aber auch um eigenständige Tumorentitäten wie z. B. epitheloide Sarkome oder alveoläre Weichteilsarkome.

Da neben diesen hochmalignen Sarkomen auch seltene benigne Tumoren (Granularzelltumor) oder selbstlimitierende pseudosarkomatöse Prozesse (proliferative Myositis) sich als überwiegend aus epitheloiden Zellen bestehende Veränderung präsentieren können, ist bei der
Dignitätsbeurteilung Vorsicht geboten und die klinische Korrelation notwendig. Bei diesem morphologischen Erscheinungsbild ist es besonders wichtig, eine Karzinom-, Mesotheliom- bzw. Melanommetastase auszuschließen.

\section{Differenziertes Gewebe}

Die geweblichen Bestandteile der Läsionen aus dieser Gruppe sind zytomorphologisch einfach zu erkennen: Man findet Fragmente von reifem Fett- bzw. hyalinem Knorpelgewebe (• Abb. 6). Bei den lipomatösen Läsionen handelt es sich in der überwältigenden Mehrheit der Fälle um oberflächlich lokalisierte Knoten, die klinisch benignen Lipomen entsprechen.
Die Punktion erfolgt häufig auf Wunsch der Patienten, bei Größenzunahme bzw. zum Ausschluss von Metastasen bekannter Neoplasien.

Die Abgrenzung zwischen unbedenklichen Lipomen und atypischen lipomatösen Tumoren/gut differenzierten Liposarkomen kann vor allem bei tief gelegenen Läsionen wegen der kleinherdigen Fettgewebsnekrosen mit reaktiven Atypien erschwert sein. Bei den im Schulter-Nacken-Bereich gelegenen lipomatösen Veränderungen mit Atypien muss man differenzialdiagnostisch die gutartigen Spindelzell- und pleomorphzelligen Lipome in Betracht ziehen, ehe man einen Malignitätsverdacht äußert. 


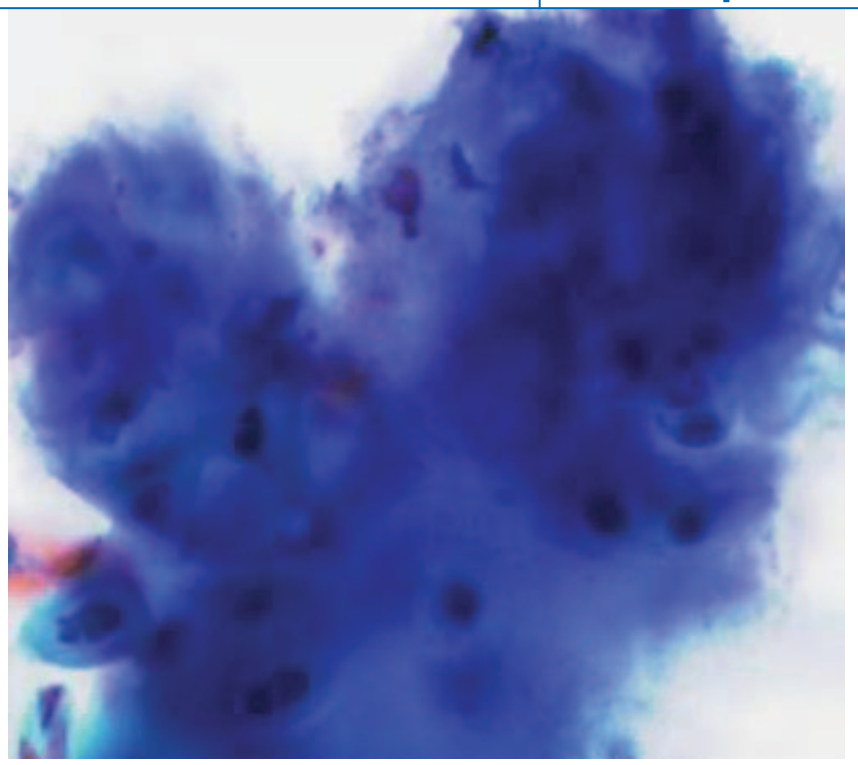

Abb. $6 \Delta$ Der 76-jährige Patient, bei welchem 2 Jahre zuvor ein Plattenepithelkarzinom der Lunge diagnostiziert und operiert wurde, bemerkte eine wachsende, $8 \mathrm{~cm}$ große Schwellung des Schulterblatts. Die FNP des Skapulaknotens ergab zellarme Fragmente von hyalinem Knorpelgewebe. Angesichts der Lokalisation und des Alters des Patienten wurde die Diagnose eines konventionellen Chondrosarkoms gestellt, was im Resektat bestätigt wurde

\section{Bedeutung von Zusatzuntersuchungen}

Der Zytopathologe ist im Grunde mit dem gleichen Gewebe und den gleichen Zellen konfrontiert wie der Histopathologe, der Stanzbiopsien bzw. offene Biopsien beurteilt. Bis auf einige technische Einzelheiten stehen ihm grundsätzlich die gleichen Zusatzuntersuchungen zur Verfügung $[10,11,15,17]$. Insbesondere der Einsatz der Zellblocktechnik lässt den scheinbaren Widerspruch zwischen einer FNP und einer Stanzbiopsie in vielen Fällen schwinden - die Bearbeitung, die diagnostischen Kriterien und die zur Verfügung stehenden Methoden sind identisch [30, 32]. In Bezug auf bestimmte diagnostische Methoden, vor allem die Fluoreszenz-insitu-Hybridisierung (FISH), ist jedoch der zytologische Ausstrich zu bevorzugen, da die schnittbedingten Artefakte eines histologischen Schnitts im dreidimensionalen Ausstrich entfallen.

\section{Grenzen der Zytopathologie}

Der hohe Stellenwert der FNP in der Diagnostik von Rezidiven bzw. Metastasen klinisch bekannter Tumoren der Weichteile ist unbestritten. Bei der Diagnostik primärer Weichteilneoplasien ist jedoch die Zytologie mit Einschränkungen konfrontiert, die auf die inhärenten Eigenschaften der untersuchten Läsionen zurückzuführen sind. Die stark kollagenhaltigen, zellarmen primären Weichteilläsionen, wie z. B. Fibromatosen oder niedrig maligne Sarkome, ergeben in einer FNP naturgemäß nur wenig Gewebe [8], während proliferative pseudosarkomatöse Prozesse vom Typ der nodulären Fasziitis recht zellreich sein können sowie zahlreiche Mitosen und ausgeprägte reaktive Atypien aufweisen [4, 33]. Die spezifische Diagnose und präzise Dignitätseinschätzung kann in solchen Fällen schwierig sein, was jedoch nicht nur auf die zytologischen Proben, sondern auch für Stanzbiopsien zutrifft. Die morphologische Beurteilung solcher Proben erfordert eine sorgfaltige Korrelation mit dem klinischen Verlauf und der Bildgebung.

Ähnlich wie die Stanzbiopsie oder sogar die Inzisionsbiopsie unterliegt die FNP dem Risiko eines „sampling error“, was zu einer Fehleinschätzung der Dignität führen kann. Die histologische Gradierung der Malignität eines Sarkoms gehört zu den wichtigsten prognostischen Faktoren. Die zytologisch durchgeführte Gradierung der Weichteilsarkome (unter
Berücksichtigung der Kerneigenschaften, Mitoserate und der Tumornekrose) korreliert gemäß einigen Studien gut mit der histologischen Gradierung gemäß FNCLCC-System mit Grad-Konkordanz bis $74 \%[26,27,29]$. Problematisch sind jedoch methodenbedingte Artefakte, vor allem in Bezug auf die Einschätzung der Mitoserate oder der Ausdehnung der Nekrose. Der Stellenwert der zytologischen Gradierung wurde noch nicht in vielen Studie belegt.

Zusammenfassend sind nicht nur die epithelialen Neoplasien, sondern auch die primären Weichteiltumoren einer zytologischen Diagnostik zugänglich. Ein multidisziplinärer Ansatz erlaubt die gezielte Anwendung moderner diagnostischer Methoden. Optimale Ergebnisse können erreicht werden, wenn der Zytopathologe entweder bei der Materialentnahme anwesend ist oder die FNP selbst durchführt. Die Diskussion mit dem Orthopäden und/oder Radiologen liefert ihm Anhaltspunkte zur Differenzialdiagnose, die gestützt auf den konventionellen zytologischen Befund und untermauert durch die molekularen Zusatzmethoden zu einer klinisch relevanten Aussage (Diagnose) führen können.

\section{Fazit für die Praxis}

Die Feinnadelpunktion (FNP) stellt eine einfache, kostengünstige und komplikationsarme Methode in der Abklärung raumfordernder Prozesse der Weichteile dar. Zytomorphologisch lassen sich in den meisten Fällen entzündliche Veränderungen, Metastasen von Karzinomen und Melanomen bzw. lymphoproliferative Prozesse abgrenzen. Bei primär mesenchymalen Läsionen erlaubt eine Kombination von Zytologie mit modernen Zusatztechniken sowie den klinischen und radiologischen Befunden in vielen Fällen eine präzise Subtypisierung eines Tumors. Eine Unterscheidung zwischen benignen und malignen Läsionen ist bei adäquaten Proben fast immer möglich. Entscheidend für die Aussagekraft der FNP eines Weichteilprozesses sind eine hohe Qualität des Untersuchungsmaterials, Kenntnisse des Zytopathologens über die differenzialdiagnostischen Möglichkeiten und eine enge, 
multidisziplinäre Zusammenarbeit mit dem Orthopäden, Radiologen und Onkologen.

\section{Korrespondenzadresse \\ PD Dr. B. Bode-Lesniewska}

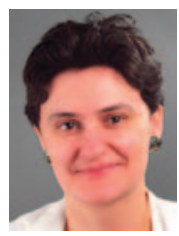

Institut für Klinische Pathologie, UniversitätsSpital Zürich

Schmelzbergstr. 12,

8032 Zürich

Schweiz

beata.bode@usz.ch

Danksagung. Die molekulargenetischen Analysen werden dankenswerterweise im Labor von Prof. Dr. D. Zimmerman, Institut für Pathologie, Universitätsspital Zürich durchgeführt.

Interessenkonflikt. Die korrespondierende Autorin gibt an, dass kein Interessenkonflikt besteht.

\section{Literatur}

1. Akerman M, Domanski HA (2003) The cytology of soft tissue tumours. Karger, New York

2. Colin P, Lagace R, Caillaud JM et al (2010) Fineneedle aspiration in myxofibrosarcoma: experience of Institut Curie. Diagn Cytopathol 38:343-346

3. Das K, Hameed M, Heller D et al (2003) Liquid-based vs. conventional smears in fine needle aspiration of bone and soft tissue tumors. Acta Cytol 47:197-201

4. Dodd LG, Martinez S (2001) Fine-needle aspiration cytology of pseudosarcomatous lesions of soft tissue. Diagn Cytopathol 24:28-35

5. Domanski HA (2005) FNA diagnosis of dermatofibrosarcoma protuberans. Diagn Cytopathol 32:299-302

6. Domanski HA, Akerman M, Carlen B et al (2005) Core-needle biopsy performed by the cytopathologist: a technique to complement fine-needle aspiration of soft tissue and bone lesions. Cancer 105:229-239

7. Domanski HA, Akerman M, Rissler P et al (2006) Fine-needle aspiration of soft tissue leiomyosarcoma: an analysis of the most common cytologic findings and the value of ancillary techniques. Diagn Cytopathol 34:597-604

8. Domanski HA, Mertens F, Panagopoulos I et al (2009) Low-grade fibromyxoid sarcoma is difficult to diagnose by fine needle aspiration cytology: a cytomorphological study of eight cases. Cytopathology 20:304-314

9. Fletcher CDM, Unni KK, Mertens F (2002) (Hrsg) Tumours of soft tissue and bone. IARC, Lyon

10. Frostad B, Tani E, Brosjo $O$ et al (2002) Fine needle aspiration cytology in the diagnosis and management of children and adolescents with Ewing sarcoma and peripheral primitive neuroectodermal tumor. Med Pediatr Oncol 38:33-40

11. Gautam U, Srinivasan R, Rajwanshi A et al (2010) Reverse transcriptase-polymerase chain reaction as an ancillary molecular technique in the diagnosis of small blue round cell tumors by fine-needle aspiration cytology. Am J Clin Pathol 133:633-645
12. Geisinger KR, Abdul-Karim FW (2008) Fine needle aspiration biopsy of soft tissue tumors. In: Weiss SW, Goldblum JR (Hrsg) Enzinger and Weiss's soft tissue tumors. Mosby Elsevier, St. Louis, S 103-117

13. Jakowski JD, Mayerson J, Wakely PE (2010) Fineneedle aspiration biopsy of the distal extremities: a study of 141 cases. Am J Clin Pathol 133:224-231

14. Kaffenberger BH, Wakely PE Jr, Mayerson JL (2010) Local recurrence rate of fine-needle aspiration biopsy in primary high-grade sarcomas. J Surg Oncol 101:618-621

15. Kilpatrick SE, Bergman S, Pettenati MJ et al (2006) The usefulness of cytogenetic analysis in fine needle aspirates for the histologic subtyping of sarcomas. Mod Pathol 19:815-819

16. Kilpatrick SE, Ward WG, Bos GD (2000) The value of fine-needle aspiration biopsy in the differential diagnosis of adult myxoid sarcoma. Cancer 90:167-177

17. Kilpatrick SE, Ward WG, Cappellari JO et al (1999) Fine-needle aspiration biopsy of soft tissue sarcomas. A cytomorphologic analysis with emphasis on histologic subtyping, grading, and therapeutic significance. Am J Clin Pathol 112:179-188

18. Klijanienko J, Caillaud JM, Lagace R (2004) Fineneedle aspiration in liposarcoma: cytohistologic correlative study including well-differentiated, myxoid, and pleomorphic variants. Diagn Cytopathol 30:307-312

19. Klijanienko J, Caillaud JM, Lagace R et al (2002) Cytohistologic correlations in 56 synovial sarcomas in 36 patients: the Institut Curie experience. Diagn Cytopathol 27:96-102

20. Klijanienko J, Caillaud JM, Lagace R et al (2003) Cytohistologic correlations in angiosarcoma including classic and epithelioid variants: Institut Curie's experience. Diagn Cytopathol 29:140-145

21. Klijanienko J, Caillaud JM, Lagace R et al (2002) Cytohistologic correlations of 24 malignant peripheral nerve sheath tumor (MPNST) in 17 patients: the Institut Curie experience. Diagn Cytopathol 27:103-108

22. Klijanienko J, Caillaud JM, Lagace R et al (2003) Fine-needle aspiration of leiomyosarcoma: a correlative cytohistopathological study of 96 tumors in 68 patients. Diagn Cytopathol 28:119-125

23. Layfield LJ (2002) Cytopathology of bone and soft tissue tumors. Oxford University Press, Oxford

24. Layfield LJ, Dodd LG, Hirschowitz S et al (2010) Fine-needle aspiration of primary osseous lesions: $A$ cost effectiveness study. Diagn Cytopathol 38:239243

25. Maitra A, Ashfaq R, Saboorian MH et al (2000) The role of fine-needle aspiration biopsy in the primary diagnosis of mesenchymal lesions: a community hospital-based experience. Cancer 90:178-185

26. Mallik MK, Dey P, Gupta SK et al (2010) Grading of soft tissue sarcomas on fine-needle aspiration cytology smear. Diagn Cytopathol 38:109-112

27. Mathur S, Kapila K, Verma K (2003) Accuracy of cytological grading of spindle-cell sarcomas. Diagn Cytopathol 29:79-83

28. Ng VY, Thomas K, Crist M et al (2010) Fine needle aspiration for clinical triage of extremity soft tissue masses. Clin Orthop Relat Res 468:1120-1128

29. Palmer HE, Mukunyadzi P, Culbreth W et al (2001) Subgrouping and grading of soft-tissue sarcomas by fine-needle aspiration cytology: a histopathologic correlation study. Diagn Cytopathol 24:307316
30. Pfeiffer DF, Bode-Lesniewska B (2006) Fine needle aspiration biopsy diagnosis of angiosarcoma after breast-conserving therapy for carcinoma supported by use of a cell block and immunohistochemistry. Acta Cytol 50:553-556

31. Wakely PE Jr, Bos GD, Mayerson J (2005) The cytopathology of soft tissue mxyomas: ganglia, juxtaarticular myxoid lesions, and intramuscular myxoma. Am J Clin Pathol 123:858-865

32. Wakely PE Jr, Kneisl JS (2000) Soft tissue aspiration cytopathology. Cancer 90:292-298

33. Wong NL (2002) Fine needle aspiration cytology of pseudosarcomatous reactive proliferative lesions of soft tissue. Acta Cytol 46:1049-1055 